\title{
Has kinesio tape a thermal effect on sprint cycling performance? A thermographic study.
}

\author{
by D. Formenti ${ }^{*}$ N. Ludwig** ${ }^{* *}$ A. Trecroci*, A. Rossi ${ }^{*}$, I. Fernandez-Cuevas ${ }^{* * *}$, M. Gargano**, \\ A. Caumo*, and G. Alberti* \\ * School of Exercise Sciences, Department of Biomedical Sciences for Health, \\ Università degli Studi di Milano, Italy. damiano.formenti@unimi.it \\ ** Department of Physics, Università degli Studi di Milano, Italy. \\ nicola.ludwig@unimi.it \\ *** Department of Sports, Faculty of Sciences for Physical Activity and Sport (INEF), \\ Universidad Politécnica de Madrid, Spain.ismael.fernandez@upm.es
}

\begin{abstract}
The aim of this study was to investigate the immediate effect that kinesio taping (KT) application on vastus lateralis and vastus medialis has on skin temperature (Tsk) of rectus femoris before and after a sprint cycling effort. Eight subjects were tested on a $6 \mathrm{~s}$ cycling sprint in two conditions: without KT (nKT), and with KT on both limbs (KT2). Thermal images of thighs were recorded before and after each sprint, thus providing skin temperature variations of rectus femoris $(\Delta T)$. Performance outcomes were Peak power (PP), Average power (AP) and Total work, recorded during the $6 \mathrm{~s}$ cycling sprint. PP and $\Delta T$ were significantly higher in the KT2 with respect to the nKT condition $(p<0.05)$. This may be attributed to a thermal effect produced by the KT.
\end{abstract}

\section{Introduction}

Kinesio taping (KT) is a method used to produce therapeutic effects in rehabilitation in sports medicine, and also used by athletes of various disciplines. Recently, it has been hypothesized that KT application can enhance muscle power and strength performance [1]. Several studies demonstrated its efficacy in increasing isokinetic and isotonic function $[2,3]$. However, other studies failed to find beneficial effect during a strength effort [4-6].

The mechanisms by which KT should enhance power and strength performance have not been completely clarified yet. Authors supposed that KT may produce a tactile stimulation on the skin, thus activating mechanoreceptors located in the dermis. Due to the material properties of KT, it is very likely that its application produces an increment in skin temperature (Tsk) of the body area close to those area where the kinesio tape is applied.

The use of infrared thermography in the study of human thermoregulation associated to physical exercise is increasing [7-10]. Recently, Priego Quesada and colleagues conducted a study for investigating the effect of graduated compressive stockings on skin temperature in runners using infrared thermography [8]. Although the condition with the garments did not produce a significant change in heart rate and perception of fatigue, it was found that running with graduated compressive stockings induced an increase in skin temperature not only in body regions that were in contact with the garments, but also in those regions of the thigh that were not [8].

Based on these observations, it is legit to hypothesize that the possible improvement in muscle power and strength performance induced the kinesio taping application may be the result of a thermal effect, similar to those found by Priego Quesada [8]. However, whether, when, and why KT has positive effect on muscle performance have not been completely investigated.

Therefore, the aim of the present study was to investigate the immediate effect of kinesio taping application on vastus lateralis and vastus medialis on Tsk of rectus femoris by infrared thermography [7] before and after a sprint performance on a cycle ergometer. It was hypothesized that the kinesio taping application would result in an increment of the skin temperature of the rectus femoris, probably reflecting an increment in sprint performance.

\section{Materials and Methods}

\subsection{Subjects}

Eight physically active males participated voluntarily in this study (age: $23.5 \pm 1.7$ years; body mass: $70.3 \pm 8.1$ $\mathrm{kg}$; height: $1.79 \pm 0.9 \mathrm{~m}$ ). They had no history of musculoskeletal injury in the lower limbs for the year before the initiation 


\subsection{1/qirt.2016.035}

of the study. Participants were informed about the protocols and procedures before their confirmation, and they provided a written informed consent. The study was approved by the Ethical Committee of the Università degli Studi di Milano.

\subsection{Procedures}

After two familiarization sessions the protocol was structured in two experimental trials (5 days between each other), which involved a single $6 \mathrm{~s}$ sprint on a cycle ergometer (Monark 894E). Participants were tested randomly in two different conditions: without taping (nKT), and with the tape applied on both legs (KT), during which Peak Power (PP), Mean Power (MP) and Total Work (TW) were recorded. Standard two-inch $(5 \mathrm{~cm})$ black kinesio taping (KT tex gold $B)$ was applied by the same certified practitioner on vastus lateralis and vastus medialis muscles longitudinally in the KT condition. In both experimental trials, athletes in swimsuit performed a warm-up at $100 \mathrm{~W}$ for 4 min on the cycle ergometer. After that, they rested for $15 \mathrm{~min}$ to acclimate to the room environmental condition $\left(22-23^{\circ} \mathrm{C} ; 50 \pm 5 \%\right)$.

Skin temperature of the muscle quadriceps body area was measured by infrared thermography. Thermal images were recorded immediately before and after the $6 \mathrm{~s}$ sprint bouts using a 14-bit digital infrared thermal camera (AVIO, TVS-700, $320 \times 240$ Microbolometric Array; 8-14 $\mu \mathrm{m}$ spectral range; $0.07{ }^{\circ} \mathrm{C}$ thermal resolution). The subjects were advised to abstain from alcohol and caffeinated drinks, and to refrain from physical activity involving lower limbs 24 $\mathrm{h}$ before the tests due to the potential influence on Tsk [11]. Skin surface was prepared by removing hairs at least 24 hours before the tests.

\subsection{Data analysis and statistics}

To obtain Tsk outcomes, thermal images were analyzed using GRAYESS ${ }^{\circledR}$ IRT Analyzer, Version 4.8. A region of interest $(\mathrm{ROI})$ corresponding to the rectus femoris was selected manually by an expert operator, thus averaging all the temperature pixels within each ROI (Figure 1). Finally, a unique temperature value for each subject was calculated as the mean of right and left ROI temperatures at each time point. Emissivity value was set to 0.98 .

Skin temperature was higher after sprint exercise with respect to the basal. Therefore, temperature variation $(\Delta \mathrm{T})$ was calculated as the difference between temperature immediately after and before each sprint (expressed in ${ }^{\circ} \mathrm{C}$ ) [9]. The normality of the data distribution was checked by graphical method and by the Shapiro Wilk's Normality Test. All the data met the assumption of normality, and therefore parametric statistics was used. $\triangle T, P P, A P$ and TW was compared between nKT and KT2 conditions using a paired sample t-test. A p-value lower than 0.05 was considered statistically significant.

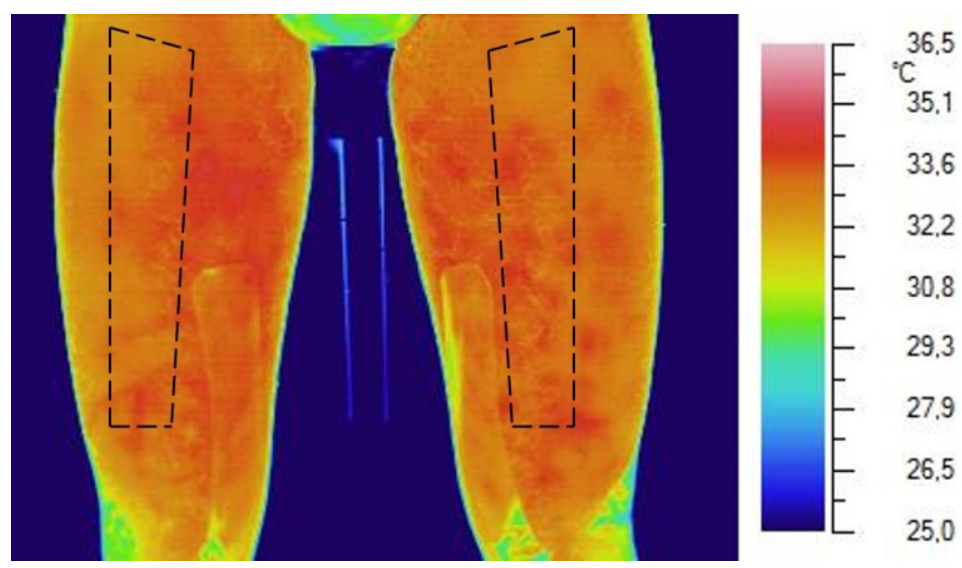

Fig. 1. A representative thermal image of one subject with the region of interests on the rectus femoris in the KT2 condition. It is possible to notice the presence of kinesio taping, especially on the vastus medialis.

\section{Results}

Mean values and standard deviations of PP and AP are shown in the graphs of Figure 1. Mean values and standard deviations of TW and $\Delta \mathrm{T}$ are shown in the graphs of Figure 2.

PP was significantly higher in the KT2 condition with respect to the nKT condition $(p=0.04) . \Delta T$ was significantly higher in the KT2 condition with respect to the nKT condition $(p<0.01)$. AP and TW were similar in the two conditions $(p=0.15$ and $p=0.3$, respectively). 

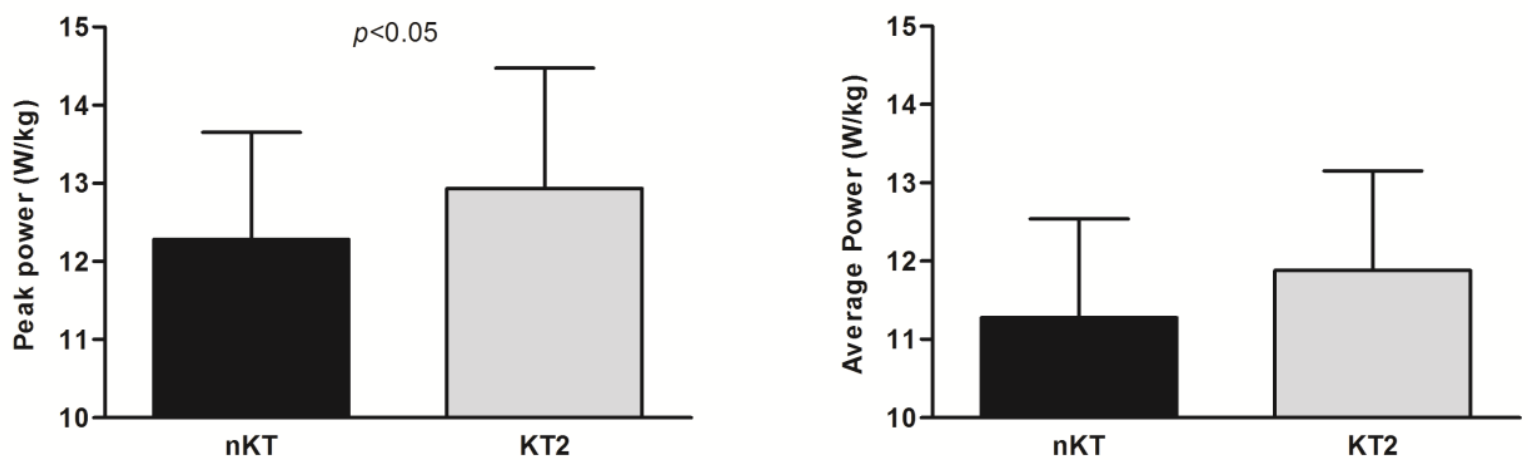

Fig. 2. Mean values of $P P, A P$, in $n K T$ and $K T 2$ conditions. Error bars are standard deviations.
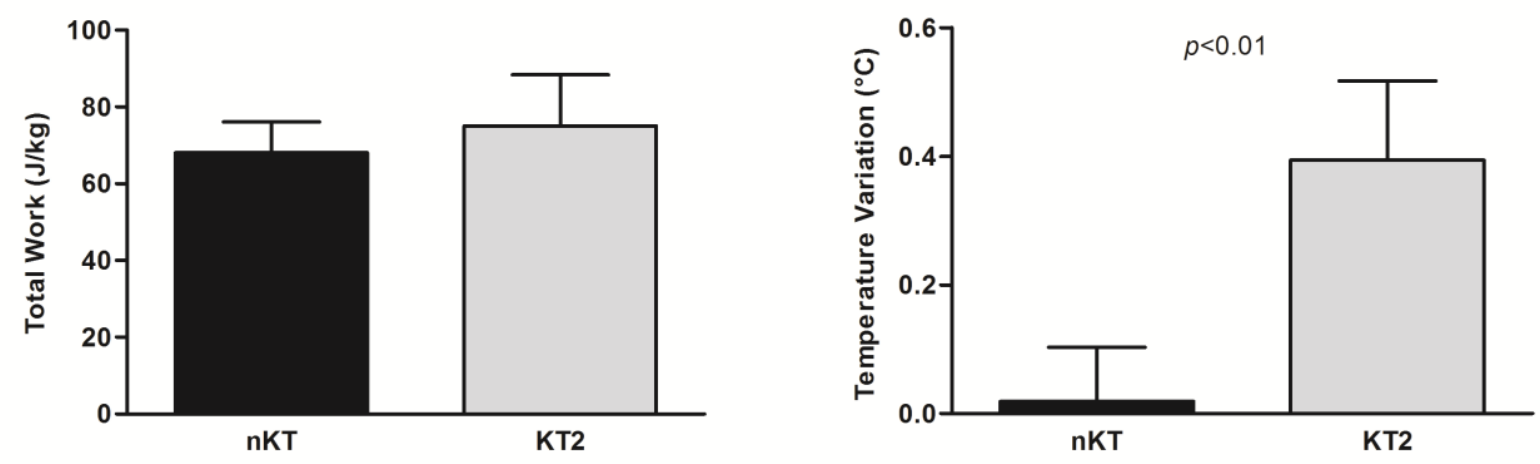

Fig. 3. Mean values of TW and $\Delta T$ in $n K T$ and $K T 2$ conditions. Error bars are standard deviations.

\section{Discussion}

The main finding of this study is that the application of KT on vastus lateralis and vastus medialis enhanced sprint cycling performance. The increment in PP reflected an increment in $\Delta \mathrm{T}$ of the rectus femoris, and viceversa (see Figure 2 and Figure 3).

To the best of our knowledge, this is the first study that have investigated the acute effect of KT application on vastus lateralis and vastus medialis on skin temperature of rectus femoris, before and after a $6 \mathrm{~s}$ cycling sprint performance. We wanted to test the possible thermal effect of KT on the performance of cycling sprint, using infrared thermography. Our hypothesis that the kinesio taping application would have resulted in an increment of the skin temperature of the rectus femoris reflecting an increment in performance was verified.

The positive effect of KT application on sprint cycling performance (30 s Wingate test) was previously observed by other authors [12]. However, another study failed to find a significant effect of KT [13].

From a speculative point of view, our positive outcomes may be attributed to an occurred thermal effect on skin temperature of thighs' anterior surface, as previously found by Priego Quesada [8]. In fact, temperature variation of rectus femoris was higher in the KT2 compared to the nKT condition. However, the increment in PP was modest, and no significant improvements were found for AP and TW.

Further studies are required to corroborate our results, and to assess if the possible thermal effects of kinesio taping application are related to an increment in skin blood flow. 


\section{REFERENCES}

[1] Fratocchi, G., Di Mattia, F., Rossi, R., Mangone, M., et al., Influence of Kinesio Taping applied over biceps brachii on isokinetic elbow peak torque. A placebo controlled study in a population of young healthy subjects. $J$. Sci. Med. Sport. 2013, 16, 245-249.

[2] Vithoulka, I., Beneka, A., Malliou, P., Aggelousis, N., et al., The effects of Kinesio-Taping® on quadriceps strength during isokinetic exercise in healthy non athlete women. Isokinet. Exerc. Sci. 2010, 18, 1-6.

[3] Huang, C.-Y., Hsieh, T.-H., Lu, S.-C., Su, F.-C., Effect of the Kinesio tape to muscle activity and vertical jump performance in healthy inactive people. Biomed. Eng. Online. 2011, 10, 70.

[4] Fu, T.-C., Wong, A.M.K., Pei, Y.-C., Wu, K.P., et al., Effect of Kinesio taping on muscle strength in athletes-a pilot study. J. Sci. Med. Sport. 2008, 11, 198-201.

[5] Nunes, G.S., de Noronha, M., Cunha, H.S., Ruschel, C., Borges, N.G., Effect of kinesio taping on jumping and balance in athletes: a crossover randomized controlled trial. J. Strength Cond. Res. 2013, 27, 3183-3189.

[6] Vercelli, S., Sartorio, F., Foti, C., Colletto, L., et al., Immediate effects of kinesiotaping on quadriceps muscle strength: a single-blind, placebo-controlled crossover trial. Clin. J. Sport Med. 2012, 22, 319-326.

[7] Formenti, D., Ludwig, N., Gargano, M., Gondola, M., et al., Thermal Imaging of Exercise-Associated Skin Temperature Changes in Trained and Untrained Female Subjects. Ann. Biomed. Eng. 2013, 41, 863-871.

[8] Priego Quesada, J.I., Lucas-Cuevas, A.G., Gil-Calvo, M., Giménez, J.V., et al., Effects of graduated compression stockings on skin temperature after running. J. Therm. Biol. 2015, 52, 130-136.

[9] Priego Quesada, J.I., Martínez, N., Palmer, R.S., Psikuta, A., et al., Effects of the cycling workload on core and local skin temperatures. Exp. Therm. Fluid Sci. Accepted and in press.

[10] Formenti, D., Ludwig, N., Trecroci, A., Gargano, M., et al., Dynamics of thermographic skin temperature response during squat exercise at two different speeds. J. Therm. Biol. Accepted and in press.

[11] Fernández-Cuevas, I., Bouzas Marins, J.C., Arnáiz Lastras, J., Gómez Carmona, P.M., et al., Classification of factors influencing the use of infrared thermography in humans: A review. Infrared Phys. Technol. 2015, 71, 2855.

[12] Dae-Young, K., Byoung-Do, S., Immediate Effect of Quadriceps Kinesio Taping on the Anaerobic Muscle Power and Anaerobic Threshold of Healthy College Students. J. Phys. Ther. Sci. 2012, 24, 919-923.

[13] Harmanci, H., Kalkavan, A., Karavelioglu, M., Yuksel, O., et al., Effects of kinesio taping on anaerobic power and capacity results. J. Sports Med. Phys. Fitness. 2015, Apr, 30. 